\title{
DETERMINATION OF REFERENCE INTERVAL OF LIVER FUNCTION TESTS DURING PREGNANCY IN URBAN AREA OF DISTRICT RAWALPINDI PAKISTAN
}

\author{
Alveena Younas, Mehwish Gilani, Qurat Ul Ain, Naveed Asif, Muhammad Aamir, Asif Ali \\ Armed Forces Institute of Pathology/National University of Medical Sciences (NUMS) Rawalpindi Pakistan
}

\begin{abstract}
Objective: To determine the reference Interval of liver function tests during uncomplicated pregnancy in population of Rawalpindi and assess their correlation over first and second trimesters of pregnancy.

Study Design: Cross sectional study.

Place and Duration of Study: Department of Chemical Pathology \& Endocrinology Armed Forces Institute of Pathology Rawalpindi from Feb 2017 to Jun 2018.

Methodology: Seven hundred and fifty four pregnant women with uncomplicated, single intrauterine pregnancy were recruited from Rawalpindi. Thirteen patients with known history of Diabetes Mellitus, hypertension, liver disease, renal disorders and those on anti-epileptics, non-steroidal anti-inflammatory drugs and steroids were excluded from the study. Blood sample was taken from each subject to analyze serum bilirubin, albumin, total alkaline phosphates and alanine aminotransferase on random access discrete auto analyser, ADVIA 1800 Chemistry system manufactured in Japan for Siemens Healthcare Diagnostics Inc. Data for serum bilirubin, albumin, ALP and ALT were expressed as mean \pm standard deviation. As the reference data followed the Gaussian distribution, therefore the 2.5th and 97.5th percentiles were estimated by values approximately 2SD on each side of mean. Results: After analysis of serum samples of 754 subjects, the reference intervals for bilirubin, albumin, ALP and ALT during first trimester were; bilirubin 3-9 $\mu \mathrm{mol} / \mathrm{l}$, albumin 31-45 g/L, ALP 122-224 U/1 and ALT 3-35 U/1,

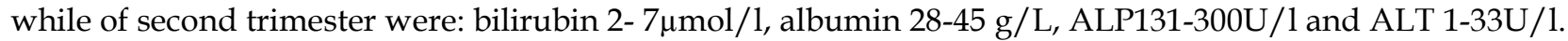

Conclusion: First and second trimester wise reference values of Liver Function Tests have been determined in pregnancy in this study. This would not only help in monitoring of normal biochemical changes of pregnancy but will also lead to prompt detection of fatal pregnancy complications.
\end{abstract}

Keywords: Liver function tests, Pregnancy, Reference interval.

This is an Open Access article distributed under the terms of the Creative Commons Attribution License (http://creativecommons.org/licenses/by/4.0), which permits unrestricted use, distribution, and reproduction in any medium, provided the original work is properly cited.

\section{INTRODUCTION}

Pregnancy is a state of physiological stress. Compensatory changes that occur during pregnancy include not only changes in energy requirements and utilization but also in different organs of maternal body such as liver to provide sufficient nurturing environment for normal growth and development of fetus ${ }^{1,2}$. Values of various analytical parameters, if compared with non-pregnant state can be erroneously labeled as abnormally low or high. Thus, it is of paramount importance that reference interval of liver function tests should be established for disease-free pregnant womenfor timely diagnosis and effective treatment of potentially fatal conditions such

Correspondence: Dr Alveena Younas, Resident Chemical Pathology, AFIP, Rawalpindi Pakistan

Received: 29 Jan 2019; revised received: 13 Jun 2019; accepted: 26 Jun 2019 as acute fatty liver of pregnancy, HELLP syndrome and Intrahepatic Cholestasis of Pregnancy ${ }^{3,4,5}$.

Liver is an integral organ of human body performing five hundred different functions. It is not only site of bilirubin, albumin and clotting factors production but also has important role in detoxification and metabolism of several substances $^{6,7}$. Various pregnancy hormones including estrogen and progesterone have effect on synthetic and metabolic functions of liver. Hepatobiliary secretion of bile and substances dissolved in it such as bilirubin, bile salts and drugs are also effected ${ }^{8-10}$. During pregnancy, plasma volume increase by $40-50 \%$ from non-gravid state with highest levels reaching towards end of second and third trimester. Thus, haemodilution leads to reduced plasma albumin levels ${ }^{11}$. A rise in serum 
alkaline phosphatase is seen in pregnancy due to increased production of both bone and placental isoenzymes ${ }^{12}$.

All these physiological changes lead to significant difference in levels of hepatic enzymes, proteins, and bilirubin, there is a substantial need of a separate reference interval for liver function tests during uncomplicated pregnancy. Only after this we would be able to monitor physiological adaptations of pregnancy and also unravel pathological conditions. This helps not only in prompt detection and management of life threatening feto-maternal liver disorders of pregnancy but also prevents undue treatment caused byfalse alarm due to normalcompensatory biochemical changes occurring in pregnancy ${ }^{13}$. Various studies have been carried out regionally and internationally by using different methods like Rank based technique, log transformation for nonparametric data and computation of mean and standard deviation for parametric data, in order to establish separate reference interval for liver function tests in pregnancy. However, local data is sparse in this regard. This study was carried out at AFIP, Rawalpindi, with an aim to determine the reference values for liver function tests for normal pregnant womenin our population and assess their correlation over first and second trimesters of pregnancy.

\section{METHODOLOGY}

This was a cross-sectional study carried out in Department of Chemical Pathology and Endocrinology, Armed Forces Institute of Pathology (AFIP), Rawalpindi from February 2017 to June 2018. Sampling technique was non-probability convenient. Seven hundred and fifty four pregnant women were recruited from local population of Rawalpindi Districtin this study. Subjects with single intrauterine, normal pregnancy were included in the study. Thirteen patients with known history of diabetes mellitus, hypertension, liver disease, renal disorders and those on anti-epileptics, non-steroidal anti-inflammatory drugs and steroid therapy were excluded from the study. The study was approved from the
Institutional Review Board (IRB) of AFIP. Samples were taken after taking informed consent from the study participants.

About $3.0 \mathrm{ml}$ of blood sample was takenfrom each subjectin yellow top gel tubeforphotometric analysis of serum bilirubin, albumin, total Alkaline phosphatase (ALP) and Alanine aminotransferase (ALT) onrandom access discrete autoanalyser, ADVIA 1800 Chemistry System, byusing Diazo (modified Jendrassik and Grof's), BCG (bromocresol green) end point; Nitro-phenyl phosphate ( $\mathrm{pNPP}$ ) Kinetic and modified IFCC (Wróblewski and LaDue) kinetic method respectively.

Data analysis was done on SPSS version 24. Kolmogorov-Smirnov test was applied to check normality of data. Data for serum bilirubin, albumin, ALP and ALT were expressed as mean \pm standard deviation (SD). As the reference data followed the Gaussian distribution, therefore the 2.5th and 97.5th percentiles were estimated by values approximately $2 \mathrm{SD}$ on each side of mean or more precisely.

$$
\begin{aligned}
& 2.5 \text { percentile }=\bar{x}-1.96 \text { SD } \\
& 97.5 \text { percentile }=\bar{x}+1.96 \text { SD }
\end{aligned}
$$

\section{RESULT}

Out of 754 participants, 396 (52.51\%) were from first trimester while three hundred and fifty eight $(47.48 \%)$ subjects were havingsecond trimester of pregnancy. Primigravida includedin the study were $302(40.05 \%)$ while 452 (59.94\%) females were multigravida. Kolmogorov Smirnov Test was applied to assess normality of data and it was found to have a normal distribution. Mean age of subjects presenting in the first and second trimester was $24.25 \pm 3.97$ years and $25.42 \pm 3.71$ years respectively (table-I). Outliers were excluded by visual inspection of data by using histogram and then statistically by using Dixon-Reed Range Test. Mean \pm standard deviation and percentiles for serum bilirubin, albumin, ALP and ALT were computed in the first and second trimesters and are shown along with the reference interval in each trimester (table-II, III). 
Table-I: Basic population characteristics.

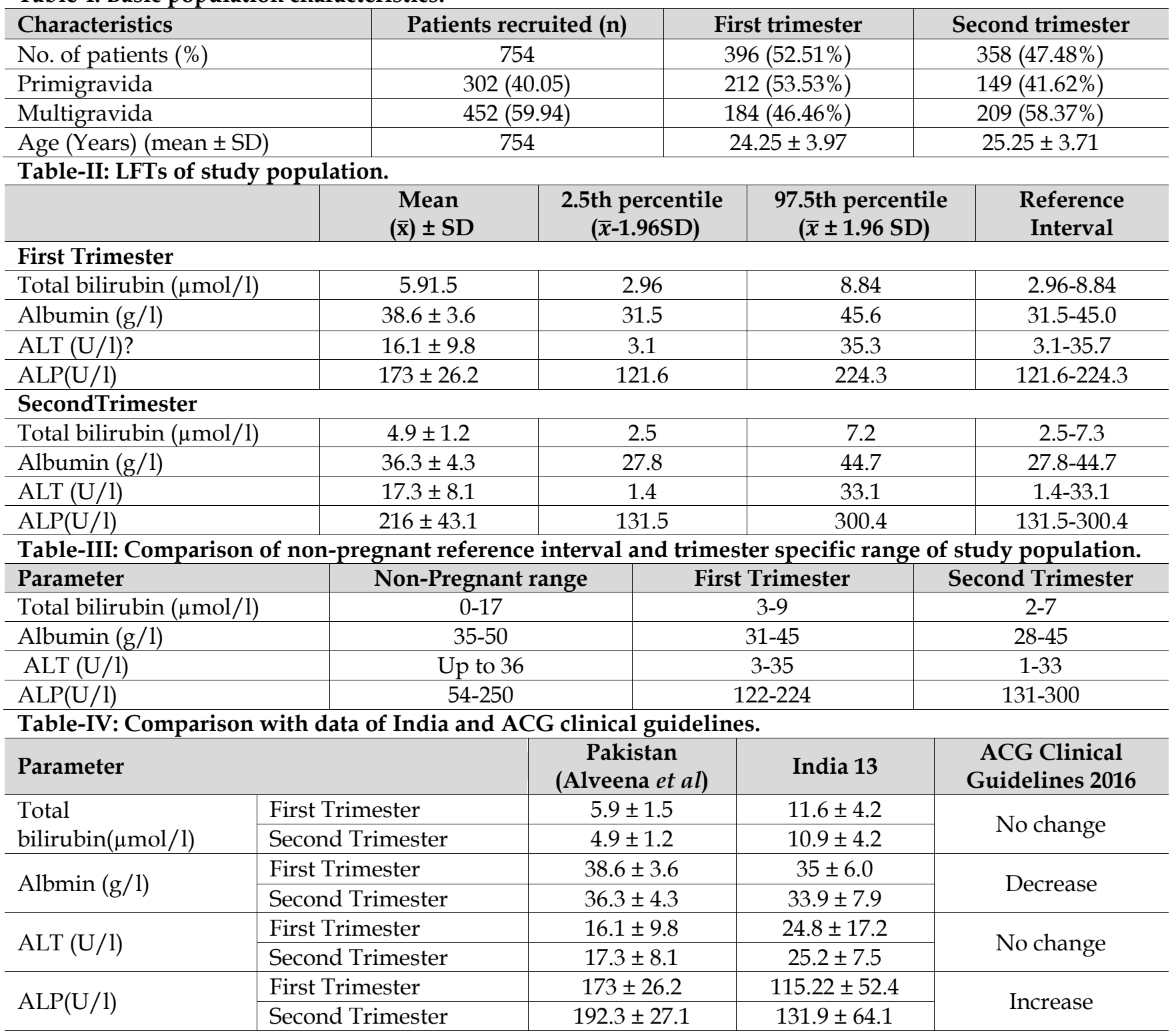

Data is expressed as mean $\pm S D$

\section{DISCUSSION}

Cardinal parameters done to assess liver function include bilirubin, hepatic enzymes such as alkaline phosphatase (ALP), Alanine aminotransferase (ALT) and albumin that reflect the state of hepaticsynthetic function ${ }^{14}$. The current study was done to analyze these parameters in healthy gravid females infirst and second trimesters.

Serum bilirubin levels were found to be lower both in first and second trimester as compared to non-pregnant levels $(0-17 \mu \mathrm{mol} / 1)$. Increase in plasma volume during pregnancy and resulting haemodilution forms logical basis of these biochemical changes. Findings of our study were in agreement with study carried out in 2016 in Gujrat (India). These lower levels of bilirubin signify the importance of separate reference interval for pregnant ladies and that even mild rise in bilirubin should be dealt with high index of suspicion requiring serial monitoring and further workup to rule out serious conditions such as acute fatty liver of pregnancy ${ }^{15}$. 
Serum albumin levels were also found to be decreased in both first and second trimester. A similar trend in albumin levels has been reported by Das et al16. Maternal plasma volume rises upto fifty percent starting from first till third trimester. Resulting haemodilution leads to lower serum albumin levels in pregnancy. However, it was observed in Das et al, that the total intravascular mass of albumin remains normal and its breakdown and formation is also not changed in comparison with non-gravid healthy controls. By establishing a lower reference interval for serum albumin in pregnancy we won't be erroneously labeling patients with hypoalbuminemia. Thus, saving them from undue treatment and stress which is harmful not only to maternal health but also can have deleterious effects to the developing fetus. Incase of hypoal-buminemia it is essential to asses albumin corrected calcium since $90 \%$ of plasma protein bound fraction of calcium is bound to albumin. Consideration of nongravid reference interval of serum albumin for a pregnant lady may lead to undue usage of albumin corrected calcium moreover diagnosis of hypocalcemia could be missed.

ALT levels did not show much variation in the first two trimesters. These findings are in agreement with ALT levels reported by previous various studies done both at regional level and internationally ${ }^{14}$. American clinical guidelines have also reported no change in serum ALT levels during all the trimesters of uncomplicated, healthy pregnancy ${ }^{13}$. Thus, increase in ALT levels during pregnancy indicate a pathological state.

Although ALP levels tend to rise both as a result of intrahepatic and extrahepatic cholestasis, yet in addition to liver it also has other sources of production including intestines, bones and placenta ${ }^{17}$. Thus ALP levels rise in pregnancy due to contribution both by placental and bone isoenzymes. Serum ALP levels were found to be on higher side in second trimester as compared to first. These findings are concurrent with results of than et al 18 , that has reported 2-4 fold rise in ALP. This makes ALP a poor marker of cholestasis especially during later stages of pregnancy. Thus, bile acids are considered better marker in cholestasis of pregnancy. So, in such conditions different fractions of ALP should be measured. Hepatic origin can also be ruled out by measuring serum gamma glutamyl transferase levels (GGT). These measures would aid the treating physicians to make an appropriate diagnosis and help in improving patient care.

The fact that our study has a good sample size along with a sensitive, precise and accurate method of analysis due to stringent monitoring of preanalytical, analytical and postanalytical factors adds up to strengths of our research. However, performing a multicentric study and inclusion of third trimester pregnant ladies would definitely yield more reliable and effective data.

\section{RECOMMEDATION}

In order to differentiate between physiological biochemical adaptations occurring in pregnancy and dangerous feto-maternal complications, laboratories should establish trimester wise reference intervals for liver function tests in pregnancy. This will help in achieving better pregnancy outcomes.

\section{Overarching Project}

The study is a part of Pakistan Society of Chemical Pathologist (PSCP) mega project of establishing reference values in general Pakistani population and special groups.

\section{Author's Contributions}

Dr Alveena Younas, sample/data collection, manuscript writing. Dr Alveena Younas, sample/ data collection, manuscript writing, Data analysis. Dr Mehwish Gilani manuscript writing, editing, Data analysis. Dr Naveed Asif study design, Manuscript editing. Dr Muhammad Aamir manuscript editing. Dr Asif Ali Manuscript editing.

\section{CONCLUSION}

First and second trimester wise reference values of Liver Function Tests have been determined in pregnancy in this study. This would not only help in monitoring of normal biochemical changes of pregnancy but will also lead to 


\section{prompt detection of fatal pregnancy compli-} cations.

\section{CONFLICT OF INTEREST}

This study has no conflict of interest to be declared by any author.

\section{REFERENCES}

1. Soma-Pillay $\mathrm{P}$, Catherine NP, Tolppanen H, Mebazaa A, Tolppanen H, Mebazaa A. Physiological changes in pregnancy. Cardiovascul J Africa 2016; 27(2): 89.

2. Gilmore LA, Butte NF, Ravussin E, Han H, Burton JH. Energy intake and energy expenditure for determining excess weight gain in pregnant women. Obstet Gynecol 2016; 127(5): 884.

3. Gabzdyl EM, Schlaeger JM. Intrahepatic cholestasis of pregnancy. J Perinatal Neonatal Nurs 2015; 29(1): 41-50.

4. Kantham L. Evaluation of biochemical parameters in hellp syndrome cases of pregnancy induced hypertension and eclampsia. J Evid Based Med Health 2015; 2(26): 3914-23.

5. Hafeez M, Ansari A, Parveen S, Salamat A, Aijaz A. Frequency of intrahepatic cholestasis of pregnancy in Punjab Pakistan: A single centre study. J Pak Med Assoc 2016; 66(2): 203-06.

6. Hamoud AR, Weaver L, Stec DE, Hinds TD. Bilirubin in the liver-gut signaling axis. Trends Endocrinol Metab 2018; 29(3): 140-50.

7. Reinke H, Asher G. Circadian clock control of liver metabolic functions. Gastroenterol 2016; 150(3): 574-80.

8. Ali DM. Effect Normal Pregnancy and Duration on Liver Enzymes Tests. Global J Med Res 2015; 15(1): 1-5.

9. Bernardi M, Ricci CS, Zaccherini G. Role of human albumin in the management of complications of liver cirrhosis. J Clin Experimen Hepatol 2014; 4(4): 302-11.

10. Dawson PA. Bile formation and the enterohepatic circulation. In Physiol Gastroint Tract (Sixth Edition) 2018; 1(1): 931-56.

11. Costantine M. Physiologic and pharmacokinetic changes in pregnancy. Front Pharmacol 2014; 5(1): 65-68.

12. Gohel MG, Joshi AG, Anand JS, Makadia JS, Kamariya CP. Evaluation of changes in liver function test in first, second and third trimester of normal pregnancy. Inter J Reproduc Contracep Obstet Gynecol 2016; 2(4): 616-20.

13. Tran TT, Ahn J, Reau NS. ACG clinical guideline: liver disease and pregnancy. Am J Gastroenterol 2016; 111(2): 176.

14. Mutua DN, Mwaniki Njagi EN, Orinda G. Liver Function Tests in Normal Pregnant Women. J Liver 2018; 7(2): 228-31.

15. Xiong HF, Liu JY, Guo LM, Li XW. Acute fatty liver of pregnancy: over six months follow-up study of twenty-five patients. World J Gastroenterol 2015; 21(6): 1927-30.

16. Das A, Teli AB, Borkotoki S. Study of Liver Function Tests in Normal Pregnancy: A Hospital Based Study. Inter J Health Scien Res 2016; 6(8): 156-60.

17. Telega GW. Hepatomegaly. In Nelson Pediatric Symptom-Based Diagnosis 2018; 1(1): 244-54.

18. Than NN, Neuberger J. Liver abnormalities in pregnancy. Best Practice Res Clinical Gastroenterol 2013; 27(4): 565-75. 